\title{
Understanding the Complexity of an Eastern Family Network
}

\author{
Shah Jamal Alam \\ Department of Computer Science, Saarland University \\ Saarbrücken, Germany
}

\begin{abstract}
Family networks have been studied by sociologists in the past and several models have been proposed. We attempt to capture the crux of eastern families systems that appears to be a fairly complex structure compared to what one finds in western families. It is important to note that by eastern systems we mean those from the South-Asian subcontinent that comprises of almost one-sixth of the world's population. The main motivation behind this work is the emergence of the field, the so-called 'complex networks'. One is interested in finding out whether the basic principles and the locality of rules that result in similar patterns could also help in understanding how this network helps in strengthening of ties among individuals in the society.
\end{abstract}

Keywords: complex networks, small-world effect, Eastern family systems, navigability.

\section{INTRODUCTION}

Over the last few years, there has been a surge of research in the study of complex networks which though appearing in many disciplines reflect surprising similarity in terms of the structural properties and dynamics. It is the similar underlying bases of such networks that have drawn the attention of researchers from quite a few disciplines. The term 'complex networks', umbrellas this inter-disciplinary and fast-growing field.

The study of complex networks dates back to the work of Paul Erdös and Alfréd Rényi [in 1950s, with their study of random graphs. The Erdös-Rényi (E-R) model provided the means for the study of complex networks for many years later. Another seminal contribution was by the sociologist Stanley Milgram whose study based on social networks led to the term 'six-degrees of separation', better known to the research community as the 'small-world' effect [1]. The term implies that in most networks (as large as the World-Wide Web), there exists a significantly shorter path between two nodes present in the E-R model which is explained in.

There have been in the recent past, phenomenal advancements in the studies of complex systems. These systems constitute of entities serving as nodes with networks forming as consequence to the interaction of nodes. The result is a vast portfolio of standard techniques for the analysis of complex networks Our purpose is not to cover such techniques here; one finds a very good literature available that cover them in detail in $[2,3]$.
Barabási [4] presents numerous fascinating examples of social networks especially the emergence of symbiosis due to the strong-ties among the nodes. A social network can be explained by means of representing the relationships existing within a community, e.g. kinship, acquaintanceship, friendship, scientific collaborations etc [5]. In terms of the analysis of complex networks, social networks differ from most non-social networks (such as the World Wide Web, Food Web etc.) due to the presence of high clustering and positive correlation between the degrees of adjacent vertices [6]. As suggested by [7], in order to have positive correlation, additional structure is required and social networks get such structure through division and groups, i.e., social networks are grouped in communities while non-social networks are not.

In this article, we explore the challenges one encounters in capturing the crux of South-Asian family systems. Studying the behavior of this network requires careful consideration as it represents the family ties with a highly complex and rich cultural heritage coupled with the fact that the region comprises almost one-sixth of the world's population, represented in almost every part of the world. The intricate nature of such network has drawn the attention of many studies by the social scientists $[8,9]$.

\section{EASTERN FAMILY NETWORK}

By an Eastern Family Network, which we shall refer as EFN in this article, we mean specifically by the family system that exists in the South Asian subcontinent. The fact that the region is a mixture of very diverse religious beliefs, ethnic backgrounds and life-styles, even though the family networks formed as result to marriages within and outside families are based on similar major rules, makes the EFN as a prospective candidate for the study of complex social networks.

\subsection{Some terminology from Social Anthropology}

Lévi-Strauss [10] presents a brilliant account to the complexity of marriage systems, especially with regards to study of marriage as a complex phenomenon governed by the rules favoring it coupled with the rules that prevent individuals from getting married.

Before discussing the marriage rules and customs which serve as the basis for the EFN, we present the basic rules of social anthropology as explained by Heady [11].

- Society is made of descents (descent groups) which continue indefinitely, and are characterized by the 
relation of 'solidarity' between their members.

- There is an incest prohibition operating at descentgroup level.

- Marriage forms patterns of exchange, which binds the different parts of a community together.

\section{Nuclear Family}

The concept of a nuclear family may vary depending upon the regional rituals. A nuclear family in our case consists of a male and female linked together taking role as husband and wife together with the sibling born to this marriage, leading to 'primary relations' between individuals namely father/mother, son/daughter, brother/sister and husband/wife.

\section{Endogamy}

With endogamy, we assume what is called the lineage endogamy in the social sciences jargon implying preferential marriages between members of same lineage as well as parallel cousin marriage. The former means marriage between cousins whose parents are siblings of different sex that is brothers and sisters; and the latter connote marriage between cousins whose parents are siblings of same sex like brothers or sisters.

\section{Exogamy, incest taboos}

The incest taboo is one of the only two social agreements found to be universal across time and culture; the second being the phenomenon of reciprocity [12]. Perhaps what has prevented closed kin to have intermarriage is the confusion that could possibly arise in maintaining the social order.

A typical eastern marriage system not only adopts the concept of endogamy, but has an important parameter, i.e., active-preference that favors marriage among cousins. This rule of cousin marriage is however constrained to the availability of matches between individuals from same generation. Ballad [9] reports that at least 50\% marriages are between cousins in the South Asian families. Important to mention is the fact, that many families do keep a fraction (though very less) of marriages that are occurred outside the family. As we shall explain later, this is an important parameter to the study of EFN.

\subsection{Rules-of-the-Game Governing EFN}

\section{Rules of Incest prohibitions and marriage restrictions}

In many western societies, first-cousin marriage is an act of incest and/or religious taboo per se. There are even a few cases in some South Asian clans which marriages between members of a nuclear family do occur. Nonetheless, we present below some of the most common exogamous and incest restrictions for an EFN in both the sociological and biological sense.
- Marriage between members of a nuclear family is prohibited; a nuclear family is therefore taken as one unit. This is common to most societies.

- Polygamy is allowed in Arab and other cultures and although a second marriage outside a society is occasionally a cause to building new relationships, we restrict single wives. There are no cases of adultery or extra-marital affairs assumed.

- In addition to nuclear family, the exogamous restrictions apply to marriages between uncle/niece, aunt/nephew, grandfather /granddaughter and grandmother/grandson.

\section{Marriage as the relation/link in the EFN}

Like most family networks, EFNs are build upon the marriage links between the individuals as the principal issue in the study of family networks is identifying the marriage-rules of marriages, and finding the right structure to represent the networks $[9,13]$. We present here some of the major rules of forming a marriage link between individuals. Marriage-links, as we shall elucidate in the subsequent section serve at different 'levels' of the EFN; at the root, forming a nuclear family, while at a higher level serving as relationships between extended families and so on. The incest and exogamous restrictions serve as 'negative' rules that curb marriages. In contrast, the positive rules are the ones that guide the formation of the linkage.

White and Houseman [14] showed that in the Turkish nomadic society, deeply influenced by the Middle Eastern culture, the marriage frequencies decline with inverse power law decay with respect to the kinship distance. Although, the South Asian kinship is quite similar in terms of inter-marriage preferences, the inverse power law follows up to a certain threshold and then the preference for choosing a marriage partner increases. The threshold is the kinship distance of an individual in search for a marriage partner. This increase of preference beyond this threshold is a striking characteristic of an EFN and is one of the important causes which explain that the kinship distance is just not at the same level of the family tree. Instead, the choice for a marriage partner in a family network takes an elliptical form, e.g. marriage of a male with his grandfather's brother's daughter is a likely incident in an EFN.

For the South Asian family structure, patrilinear descent is the guiding rule. By and large, anthropologists partition this kinship structure into two sets; one in which individuals share patrilinear descent and second affines, individuals that are related through marriages [11]. A patrilinear descent is shown in Figure 1, where $X$ and $Y$ are linked through marriage but $Y$ is at higher level than $X$. Marriage not only results in formation of cycles in the family network, such type of marriages cue strong-ties in the EFN. 
Following maybe regarded as major issues/parameters in a nutshell, central to the formation of the marriage links, which may be common as well to family systems in other societies:

- Marriage between two individuals has a much higher likelihood if they share common descent.

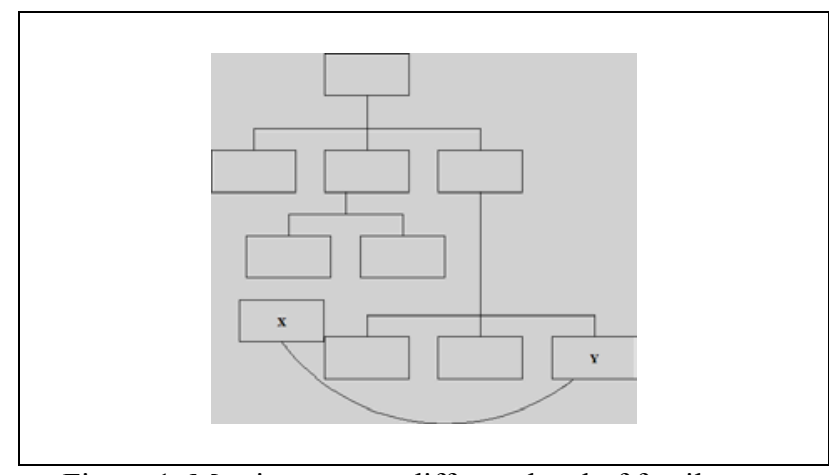

Figure 1: Marriage at two different level of family tree.

- Age Factor: Though an un-married individual in a family-network remains a candidate for marriage, one important parameter is the age. Age of a person especially a female has an important impact on the probability that an individual would be married or not.

- An upper-limit for common descent is a parameter.

- Cross Cousins Marriage: Like other social systems, formation of marriage-links prefers the cross cousin marriages.

- Watta-Satta (Cross Marriages): This is a distinctive form of marriage that applies to marriage between a husband's sister and his wife's brother, resulting in making of two linkages (marriages) simultaneously. It is preferred when an arrangement of one marriage takes place, and the siblings of the two newly wed to be, correspond to their respective matches. Interestingly, one does not come across to this rule, which is a means of strengthening ties among families both culturally and economically.

A moderate eastern marriage system not only adopts concept of endogamy, but has an important parameter active-preference that favors marriage with cousins. This rule of cousin marriage is, however, constrained to the availability of matches between individuals from same generation.

\subsection{The Desi's in the West scenario}

In the past several decades, there has been a significant influx of people from the South Asian subcontinent in the various parts of the world, especially in the West. Desi is the slang term to refer to the community that belongs to people from India, Pakistan, Bangladesh and Sri Lanka. What motivates one in analyzing the EFN is that the community is being able to preserve the structure of the family network especially since a new generation has grown up in the past decade. As families prefer to have their children married within their own community, intermarriages within the desi communities have resulted in the formation of a remarkable family network, and has resulted in high level of acquaintance among the members guided by social proximity and not spatial separation. The lesser choice for possible matches have helped lift the ethnic barrier within the cliques in the network in addition to the marriages outside the community. The highly clustered networks have been advantageous in formation of marriage links as people are more likely to find partners who are compatible in terms of the upbringing and social backgrounds.

\section{CHALLENGES IN REPRESENTING AN EFN}

A rather bigger issue in representing family networks as complex social networks is devising a proper mathematical structure to represent them. An important pre-condition in the structural representation is to represent alliance, which as a result of marriage, is formed between the families of the bride and groom, bringing members of the families closed together. This is the dynamics of family networks, as given two separate cliques, say, $X$ and $Y$ in a society, such that $\forall x \in X, y \in Y$, distance $(x, y)=\infty$, where distance represents the (shortest) path distance between two individuals. Introduction of a marriage between an $x$ and $y$ belonging to $X$ and $Y$ respectively, makes the above distance relationship finite. Alliance therefore, is defined as:

An alliance is not instituted by a simple marriage contract between man and wife but constituted upon a preexisting set of social relations, expectations, obligations, and/or privileges, existing between two groups, the givers and takers of the bride and reciprocally of the groom [13].

Building upon relationships through such marriages 'outside' the extended family, is the key in evolving a complex network and a possibly later fusion of the two cliques. A single marriage link between two cliques, is not at all adequate for this to occur; nevertheless, such marriages, are well-supported by the phenomenon of 'preferential attachment' as they increase the possibility of further possible marriages between individuals of the two cliques.

\subsection{Matching for marriage partners}

White [13] describes an attributed approach to finding out matching of possible mates in their model for family network. The technique may be extended so that for an individual in a network, a list of possible matches is kept stored.

An interesting feature in the South Asian family system is that the search for mate is not a symmetric process. Usually, it is the male for whom the marriage partner is 
searched. The problem maybe viewed as matching in a bipartite graph [15], with the choice of matches with preferences dependent upon the distance in the genealogy. As mentioned previously, preference for marriage on the basis of distance is not only at the same level in a South Asian lineage, the distance is calculated as within an ellipse. The preferential attachment dies as the distance reaches a certain radius (a parameter). However, beyond the threshold the preference grows again, where partners within a clique are chosen (who share the parentage at a much higher level).

In our proposition, we maintain a set of possible mates for an individual for every generation. The cardinality of this set should be finite and limited. Individuals who do not share common descent up till 3 generations do not belong to same family. The possibility of their marriage is then a function of number of linkages between the two families.

\subsection{A Multilevel Structure for EFN}

There have been several attempts to come out with a standard structure to be used in analyzing family networks. White [13] provides a sophisticated methodology for modeling of generalized complex family systems through the idea of relinking of individuals that are already connected in the network. As White explains, "the canonical forms of alliance, marriage rules, and marriage strategies are those individual marriages that relink families already linked".

Usually a directed graph has been used for such purpose. Traditional tree-structures for representing a family lineage are no doubt simple and straightforward. As the essence of the South Asian family system lies in the relinking, the mathematical model P-system [16], has been credited as the canonical representation [13].

A P-graph is a signed digraph is an asymmetric and acyclic with a maximum in-degree 2 (for parents); and for each arcs of each sign, the maximum in degree of nodes is 1. Starting from the nuclear family (we generate a set of nuclear families for beginning of linkages $a b$ initio), a linkage between two individuals belonging to two different nuclear families, yields another nuclear family, such that the two parent families are now linked through the marriage. For a nuclear family the structure is straightforward, as children belong to their father's genealogy and this affiliation remains while they are not married. As White and Harary [16] explain, this is the core level of the family network and in our case the structure where an individual's information is maintained. As the complexity grows, the question one needs to address is that to which family should the new nuclear family belong to? Generally a child's genealogy is basically that of their father's. While for male children, the affiliation remains after marriage, that is, the newly created nuclear family node, for females, however this affiliation changes to the family of their husbands at marriage in most cases.
P-graphs have been used in several studies of kinship network embedded with the tool Pajek [17], and have been shown to be capable of finding out important properties of the network, such as clustering, components of the graph, presence of giant-components and various patterns [16]. This article focuses not only on modeling marriage links between two individuals and families, but also how well two individuals are related to each other (or not) in a society where families are linked through marriages. What we are interested in is estimating the level of 'relationship' of two individuals picked within a network, taken into account that an individual's knowledge is limited to their closed relatives (where this notion of closed-relatives is a parameter and as mentioned above, defines the radius for the relatives' circle of an individual.

In an EFN, a link established through marriage that relates people from belonging from different families, does not break. Perhaps, the only way for a relationship between two families to be broken is divorce that breaks a nuclear family and removes all links connected to them at extended-family level of the network. However, as an EFN is strongly-tied, an occurrence of a divorce (which has still a negligible rate), does not disconnect people related to each other in the network. Even though the distance between some individuals might increase, the immensity of re-linkages does not alter significantly on average. There is need to test this social-reality by simulating such phenomenon and establish the results based on evidence.

\subsection{Navigability and Strengthening of Ties in EFN}

In recent years, it has been observed that small-worldnetworks possess two central components; a) the universality of short-chained acquaintances and b) the locality of individual's operations guide them in finding such chains [18].

As White and Houseman [14] define: The problem of navigability is whether the next step in such chains will be any closer to the target than the last.

The feature of navigability becomes more prominent in an EFN due to the marriage rules as well as the fact that not only cousin marriages are preferred but marriages at different levels of the tree are also performed, shortening the distance between two individuals greatly.

Kleinberg [18] described an approach that is based on adding long-range relationships to a lattice, controlled by a clustering coefficient that determines the probability of a connection between two nodes as a function of the lattice distance. The upshot is that efficient navigability is a fundamental property of a small-world network. For a comprehensive mathematical treatment of this approach see [19]. 


\section{CONCLUDING REMARKS}

The small-world effect and the presence of high clustering in an EFN allows members in a society to be re-linked so that the average distance between two individuals does not change very much. This effect not only guides towards reproduction of patterns in the network, they are pivotal to the flow of information (which is tremendous in an EFN) and binding of relationships among members of the society.

We are currently developing a simulation model of an EFN (which is inspired from White [13]) that emerges with agents acting as individuals in the society. Our model is expected to help in the investigations such as how a marriage give rise to a family unit in the society which stems from the families of the individual agents and how such interaction could lead to structures such as transformation of extended families into clans etc. The rules of interaction are also been fine-tuned.

\section{REFERENCES}

[1] Milgram, S., 1967, "The small world problem", Psychology Today, 2, pp. 60-67.

[2] Albert, R., and A. Barabási, 2002, "Statistical Mechanics of Complex Networks", Rev. Mod. Phys., $74,47$.

[3] Newman, M.E.J., 2001, "Small Worlds: The Structure of Social Networks", Santa Fe Institute.

[4] Barabási A., 2002, Linked: The New Science of Networks, Perseus Publishing, Cambridge, MA.

[5] Pujol, J.M., R. Sangüesa and J. Delgado, 2002, "Extracting Reputation in Multi Agent Systems by Means of Social Network Topology", in Proceedings of the AAMAS '02, ACM Press, New York.

[6] Newman, M.E.J., D.J. Watts and S.H. Strogatz, 2002, "Random graph models of social networks", in proceedings of the Natl. Acad. Sci. USA, 99, pp. 25662572 .

[7] Newman, M.E.J., and J. Park, 2003, "Why social networks are different from other types of networks", Phys. Rev. E, available at arxiv.org.

[8] White, D.R., and T. Schweizer, 1998, "Kinship, Property Transmission, and Stratification in Rural Java", in Thomas Schweizer and Douglas R. White (editors), Kinship, Networks and Exchange, pp. 36-58, Cambridge University Press.

[9] Ballad, R., 1990, "Migration and kinship: the differential effects of marriage rules on the process of Punjabi migration to Britain", in Clark, Peach, Vertovek (editors), South Asians Overseas: Context and Communities, pp. 219-249, Cambridge University Press.

[10]Lévi-Strauss, C., 1966, "The Future of Kinship Studies", in proceedings of the Royal Anthropological Institute for 1965. pp. 13-22.

[11]Heady, P., 2001, "Kinship, Courtship and Conscription: Lévi-Straussian aspect of some Alpine Village Rituals", Max Planck Institute for Social Anthropology, Halle/Saale.

[12] Gouldner, A.W., 1961, "The norm of reciprocity", American Sociological Review, 25, pp. 161-179.

[13] White, D.R., 1999, "Controlled Simulation of Marriage Systems", Journal of Artificial Societies and Social Simulation, 2 (3), available at http://www.soc.surrey.ac.uk/JASSS/2/3/5.html

[14] White, D.R., and M. Houseman, 2002, "The Navigability of Strong Ties: Small Worlds, Tie Strength and Network Topology", Complexity, 8(1), pp. 72-81.

[15] Cormen, T.H., C.E. Liverson, R.L. Rivest and C. Stein, 2002, Introduction to Algorithms, 2nd Ed., MIT Press.

[16] White, D.R., V. Batagelj and A. Mrvar, 1999, "Analyzing Large Kinship and Marriage Networks with Pgraph and Pajek", Social Science Computer Review, 17(3), pp. 245-274.

[17] Batagelj, V., and A. Mrvar, 1998, "Pajek-A Program for Large Net-works Analysis”, Connections, 21 (2), pp. 47-57.

[18] Kleinberg, J., 2000a, "Navigation in a small world", Nature, 406, pp. 845, Macmillan Magazines.

[19] Kleinberg, J., 2000b, "The small- world phenomenon: An algorithmic perspective", in proceedings of the 32nd ACM Symposium on Theory of Computing, ACM Press, New York. 